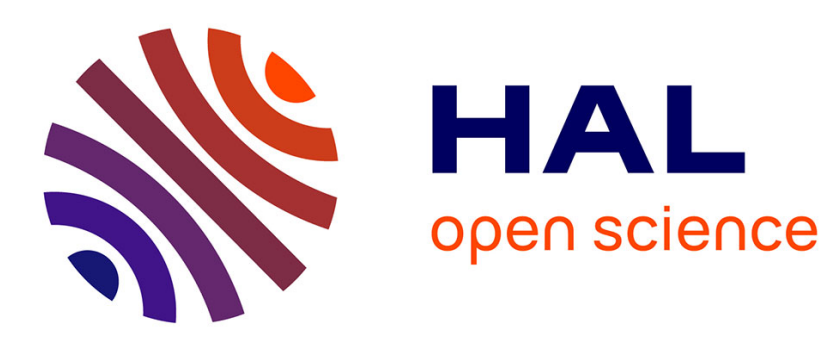

\title{
Qualité du pollen formé au froid chez la tomate (Lycopersicon esculentum Mill.). II. - Recherche de géniteurs de bonne fertilité pollinique au froid
}

Brigitte Maisonneuve

\section{- To cite this version:}

Brigitte Maisonneuve. Qualité du pollen formé au froid chez la tomate (Lycopersicon esculentum Mill.). II. - Recherche de géniteurs de bonne fertilité pollinique au froid. Agronomie, 1983, 3 (9), pp.879-882. hal-00884584

\section{HAL Id: hal-00884584 \\ https://hal.science/hal-00884584}

Submitted on 1 Jan 1983

HAL is a multi-disciplinary open access archive for the deposit and dissemination of scientific research documents, whether they are published or not. The documents may come from teaching and research institutions in France or abroad, or from public or private research centers.
L'archive ouverte pluridisciplinaire HAL, est destinée au dépôt et à la diffusion de documents scientifiques de niveau recherche, publiés ou non, émanant des établissements d'enseignement et de recherche français ou étrangers, des laboratoires publics ou privés. 


\title{
Qualité du pollen formé au froid chez la tomate (Lycopersicon esculentum Mill.). II. - Recherche de géniteurs de bonne fertilité pollinique au froid
}

\author{
Brigitte MAISONNEUVE \\ I.N.R.A., Station d'Amélioration des Plantes maraîchères, Centre de Recherches agronomiques d'Avignon, \\ B.P. 94, F 84140 Montfavet
} aucune sensibilité au traitement à $7^{\circ} \mathrm{C}$, sont les 2 lots les plus intéressants.

Mots clés additionnels : Résistance au froid, fertilité pollinique, test, Lycopersicon hirsutum, L. peruvianum, L. pimpinellifolium. pollen quality at low temperature.

\begin{abstract}
Thirty seven tomato accessions ( $28 \mathrm{~L}$. esculentum, $6 \mathrm{~L}$. pimpinellifolium, $1 \mathrm{~L}$. peruvianum, $2 \mathrm{~L}$. hirsutum $)$ were tested under controlled conditions for their ability to form pollen at low temperature (\% normal grains after a seven-day-treatment at $\left.7^{\circ} \mathrm{C}\right)$. Thirty one accessions $(26 \mathrm{~L}$. esculentum and $5 \mathrm{~L}$. pimpinellifolium $)$ were more sensitive at low temperature than the less sensitive control, 'Précoce'. Two accessions (one $L$. esculentum cv. 'Hilds matina' from Germany and one $L$. pimpinellifolium ' $108059-2(5)$ ' from U.S.S.R.) were comparable with 'Précoce'. Four accessions ( $L$. esculentum cerasiforme cv. 'Prachtlow', $L$. peruvianum sel. I.N.R.A., $2 L$. hirsutum) were less sensitive than 'Précoce'. The $2 L$. hirsutum accessions (' LA 1393' and 'LA 1777 ') originating from high altitudes in the Andes never showed any effect of the cold treatment and were therefore considered as the most interesting varieties.
\end{abstract}

Additional key words : Cold resistance, pollen fertility, germplasm, Lycopersicon hirsutum, L. peruvianum, L. pimpinellifolium.

\section{INTRODUCTION}

Il est utile de rechercher des variétés de tomate dont la fertilité pollinique est peu affectée par les basses températures. En effet, le pollen joue un rôle prédominant dans la faible nouaison au froid et des différences de sensibilité variétale ont été mises en évidence (MAISONNEUVE \& PHILOUZE, 1982). Cette recherchè est facilitée par l'utilisation d'un test en conditions contrôlées (MaISONNEUve, 1983).

La fertilité pollinique à basses températures des variétés citées dans la littérature pour leur bonne nouaison au froid n'est pas toujours connue; en effet, certains auteurs ne mentionnent pas le critère pris en compte pour juger ce caractère. D'autre part, 'dans les caractères retenus pour considérer qu'une variété noue bien à basses températures, la fertilité pollinique n'est parfois pas citée ; il en est ainsi, par exemple, pour la sélection des variétés du groupe «Sub Ärctic » effectuée au Canada (HARRIS, 1976). D'autre part, le parallélisme des réactions du pollen au froid et au chaud (ROBINSON et al., 1966) incite à tester au froid la réaction des variétés notées assez fertiles à hautes températures 
comme "Saladette» (RUDich et al., 1977). D'ailleurs plusieurs variétés sont utilisées comme géniteurs de bonne nouaison aussi bien au chaud qu'au froid ; par exemple, la variété canadienne "Sub Arctic Plenty" a été retenue en Californie comme géniteur de bonne nouaison à hautes températures (El AHMADI \& STEVENS, 1979).

Parmi les variétés de Lycopersicon esculentum notées à Montfavet pour leur bonne fertilité pollinique en serre froide, plusieurs d'entre elles ("Apéca", "Apédice", « $\mathrm{N}^{\circ} 10$ », «Précoce » et «Stupické Polni Rané ») ont pu être rattachées à un groupe variétal faisant intervenir l'espèce $L$. pimpinellifolium dans leur généalogie (MAISONNEUVE \& PHILOUZE, 1982).

Nous avons donc recherché, dans des types variétaux différents, parmi les lots cités dans la littérature pour leur bonne nouaison au froid ou au chaud ou bien originaires de régions à climat rigoureux, ceux qui se montrent au moins aussi fertiles que "Précoce».

\section{MATÉRIEL ET MÉTHODES}

\section{A. Matériel végétal}

Trente sept origines sont testées au cours de 10 expériences de 1979 à 1982 (tabl. 1) :

- 2 lignées bulgares issues de croisements avec $L$. pimpinellifolium: "Plovdiv 24-1» et «XXIV-13» (expériences $\mathrm{a}$ et $\mathrm{b}$ ).

- 6 lignées, reçues d'U.R.S.S., appartenant à l'espèce L. pimpinellifolium: « K 9738 ", « 108059-7-26 ", « K 9683 », « 108059-2-12 », « 108059-7(13)», « 108059$2(5) »$ (expérience $\mathrm{f}$ ).

- 2 origines de $L$. hirsutum trouvées à très haute altitude dans les Andes et reçues de l'Université de Davis (Californie, U.S.A.) : «LA 1393 » et «LA. 1777 » (expérience e). "LA 1777 " a déjà été mentionnée pour la bonne germination de son pollen au froid (ZAMIR et al., 1981).

- 1 origine de L. peruvianum sélectionnée à l'I.N.R.A. dans un programme de résistance au virus de la mosaïque du concombre (expérience i).

- 19 variétés de $L$. esculentum venant de régions à climat froid ou signalées pour leur bonne adaptation aux cultures en conditions froides: "NY 475 » (expérience b) de l'Université de Cornell (New York, U.S.A.) ; 5 variétés très précoces (expérience f) dont 3 de l'Université de l'Idaho (U.S.A.) : "Benewah », "Gem State», "Santa » et 2 de type cerasiforme reçues du V.I.R. de Leningrad (U.R.S.S.) : « K. 3080 », « K 342 »; "Tuckcross 756 P » (expérience $\mathrm{j}$ ), hybride 3 voies recommandé pour la culture sous serre au Canada (TRINKLEIN \& LAMBETH, 1981); 2 variétés canadiennes, "Polar Circle», intervenant dans la généalogie des variétés du type «Sub Arctic» (HARRIS, 1972) et "Sub Arctic Plenty " (expériences j et d); 1 variété hollandaise, «78 D 42 " (expérience g); 9 variétés reçues d'Allemagne : « Hilda 153 », « Hilds Matina », «Freiland früheste », «Olomoucke Nizké », "Prachtlow», «Avon Early ", "Meteor », "Outdoor Girl " et "Cudo Rynka » (expériences $\mathrm{j}, \mathrm{c}, \mathrm{d}$ et $\mathrm{f}$ ).

- 1 géniteur de bonne germination au froid, originaire de Turquie, "PI 174263 » (expérience i).

- 6 variétés de $L$. esculentum mentionnées dans des programmes d'adaptation aux hautes températures aux Etats-Unis ; «L 512-7 » de l'Université de Louisiane et "TAES 9" du Texas (expériences $h$ et i);
« Malintka 101 ", « Nagcarlan » et « Saladette » (expériences c et d) qui sont utilisées à l'Université de Davis (Californie) ainsi d'ailleurs que "Sub Arctic Plenty ", déjà mentionnée dans les variétés nouant à basses températures ; «Filipino 2 » (expérience j) qui est du type « Nagcarlan» (GRAHAM, 1961).

\section{B. Méthodes}

La méthode est celle présentée dans l'article précédent (MAISONNEUVE, 1983). Les 37 origines sont testées au cours de 10 expériences réalisées entre l'automne 1979 et le printemps 1982 (tabl. 1). Dans chaque expérience, les variétés étudiées sont comparées aux témoins, "Coldset" et «Précoce ", d'après la moyenne des mesures de qualité de pollen réalisées $5 \mathrm{j}$ consécutifs situés entre le $7^{\mathrm{c}}$ et le $17^{\circ}$ jour après la fin du traitement de $7 \mathrm{j}$ au froid.

\section{RÉSULTATS ET DISCUSSION}

Les résultats sont présentés sur la figure 1. Seize des 37 origines testées ont un pollen aussi sensible ou même plus sensible au traitement au froid que "Coldset"; 11 variétés se situent entre «Coldset » et la moyenne des 2 témoins; 6 variétés se situent entre la moyenne des 2 témoins et « Précoce » parmi lesquelles 2 sont très proches de «Précoce » (expériences $f$ et $j$ ) ; 4 origines sont supérieures à "Précoce", le témoin de bonne fertilité au froid (expériences $\mathrm{c}, \mathrm{e}, \mathrm{i}$ ).

Nous remarquons le mauvais comportement général des variétés de $L$. esculentum testées; 12 variétés sont aussi sensibles que «Coldset»: «Plovdiv 24-1», «NY 475 », «Olomoucke Nické », « Meteor », «Saladette», «Benewah", "Cudo Rynka ", "Gem State ", "Santa ", «K 3080 », « TAES 9 » et « Tuckcross 756-P » (lots $n^{\circ} 1,2$, $6,9,12,16$ à 20,32 et 37$) ; 10$ variétés sont inférieures à la moyenne des 2 témoins: "XXIV-13», « Malintka 101 », « Nagcarlan», «Sub Arctic Plenty», « K $342 »$, « 78 D 42 », «L 512-7», «PI 174263 », «Filipino 2 » et « Hilda 153 " (lots $n^{\circ} 3,5,10,13,21,28,29,31,33$ et 36). Ainsi des variétés de régions à climat rigoureux ou recommandées dans des programmes d'adaptation au chaud ou au froid ont une microsporogenèse très sensible aux basses températures. Ceci peut s'expliquer par des différences dans les conditions de tri ou des différences dans les critères d'adaptation. Par exemple, «Sub Arctic Plenty», utilisée comme géniteur de bonne nouaison au chaud, forme 75 p. 100 de fruits sans graines et son pollen formé à haute température a une très faible germination (EL AHMADI \& STEVENS, 1979). Notons que toutes les variétés testées adaptées au chaud ("Malintka 101 ", " Nagcarlan », "Saladette ", «Sub Arctic Plenty», «L 512-7», «TAES 9", «Filipino $2 »$ ) semblent avoir une mauvaise microsporogenèse à $7{ }^{\circ} \mathrm{C}$. En ce qui concerne les types variétaux, nous ne retrouvons pas, chez les lignées bulgares issues de croisements avec $L$. pimpinellifolium ("Plovdiv 24-1" et «XXIV-13»), la bonne résistance au froid notée chez d'autres variétés issues de croisements avec cette espèce comme « $\mathrm{N}^{\circ} 10$ », "Stupické Polni Rané " (MaISONNEUVE \& Philouze, 1982)

A ces 22 variétés de $L$. esculentum très sensibles au traitement à $7^{\circ} \mathrm{C}$, nous pouvons d'ailleurs joindre 5 des origines de $L$. pimpinellifolium reçues d'U.R.S.S. : «K 9738 », « 108059-7-26», « K 9683», «108059-2-12 », «108059-7 (13)» (lots $n^{\circ} 22$ à 26). 
TABLEAU 1

Expériences réalisées et variétés testées.

Experiments carried out and varieties tested.

\begin{tabular}{|c|c|c|c|c|c|c|}
\hline \multirow{2}{*}{ Expériences } & \multirow{2}{*}{$\begin{array}{c}\text { Epoque } \\
\text { du traitement }\end{array}$} & \multirow{2}{*}{$\begin{array}{l}\text { Période de } \\
\text { mesure } \\
\text { considérée }\end{array}$} & \multicolumn{2}{|c|}{ Valeurs des témoins } & \multicolumn{2}{|r|}{ Variétés étudiées } \\
\hline & & & Coldset & Précoce & $n^{\circ}$ & noms \\
\hline $\begin{array}{c}\mathrm{a} \\
(1979)\end{array}$ & déb. octobre & $11^{\mathrm{c}} \rightarrow 15^{\mathrm{e}} \mathrm{j}$ & 11 & 36 & 1 & Plovdiv 24-1 \\
\hline $\begin{array}{c}\mathrm{b} \\
(1980)\end{array}$ & mi-février & $13^{\mathrm{e}} \rightarrow 17^{\mathrm{e}} \mathrm{j}$ & 11 & 39 & $\begin{array}{l}2 \\
3\end{array}$ & $\begin{array}{l}\text { NY } 475 \\
\text { XXIV-13 }\end{array}$ \\
\hline $\begin{array}{c}\mathrm{c} \\
(1980)\end{array}$ & déb. mars & $13^{c} \rightarrow 16^{c} \mathrm{j}$ & $6(*)$ & $27(*)$ & $\begin{array}{l}4 \\
5 \\
6 \\
7\end{array}$ & $\begin{array}{l}\text { Freiland früheste } \\
\text { Malintka } 101 \\
\text { OlomouckeNizké } \\
\text { Prachtlow }\end{array}$ \\
\hline $\begin{array}{c}d \\
(1980)\end{array}$ & fin mars & $9^{c} \rightarrow 13^{e} j$ & 22 & 76 & $\begin{array}{r}8 \\
9 \\
10 \\
11 \\
12 \\
13\end{array}$ & $\begin{array}{l}\text { Avon Early } \\
\text { Meteor } \\
\text { Nagcarlan } \\
\text { Outdoor Girl } \\
\text { Saladette } \\
\text { Sub Arctic Plenty }\end{array}$ \\
\hline $\begin{array}{c}\mathrm{e} \\
(1981)\end{array}$ & déb. avril & $9^{c} \rightarrow 13^{c} j$ & 18 & 46 & $\begin{array}{l}14 \\
15\end{array}$ & $\begin{array}{l}\text { LA } 1393 \\
\text { LA } 1777\end{array}$ \\
\hline $\begin{array}{c}\mathrm{f} \\
(1981)\end{array}$ & fin mai & $7^{\mathrm{e}} \rightarrow 11^{\mathrm{e} j}$ & 35 & 53 & $\begin{array}{l}16 \\
17 \\
18 \\
19 \\
20 \\
21 \\
22 \\
23 \\
24 \\
25 \\
26 \\
27\end{array}$ & $\begin{array}{l}\text { Benewah } \\
\text { Cudo Rynka } \\
\text { Gem State } \\
\text { Santa } \\
\text { K } 3080 \\
\text { K } 342 \\
\text { K } 9738 \\
108059-7-26 \\
\text { K } 9683 \\
108059-2-12 \\
108059-7(13) \\
108059-2(5)\end{array}$ \\
\hline$\underset{(1981)}{g}$ & déb. septembre & $7^{\mathrm{e}} \rightarrow 11^{\mathrm{e}} \mathrm{j}$ & 22 & 34 & 28 & $78 \mathrm{D} 42$ \\
\hline $\begin{array}{c}\mathrm{h} \\
(1982)\end{array}$ & déb. mars & $13^{\mathrm{r}} \rightarrow 17^{\mathrm{e}} \mathrm{j}$ & 1 & 43 & 29 & L $512-7$ \\
\hline $\begin{array}{c}\mathrm{i} \\
(1982)\end{array}$ & mi-mars & $12^{\mathrm{c}} \rightarrow 16^{\mathrm{e} j}$ & 4 & 30 & $\begin{array}{l}30 \\
31 \\
32\end{array}$ & $\begin{array}{l}\text { L. peruvianum CMV sél. I.N.R.A. } \\
\text { PI } 174263 \\
\text { TAES } 9\end{array}$ \\
\hline $\begin{array}{c}j \\
(1982)\end{array}$ & $2^{\mathrm{c}}$ moitié de mars & $10^{c} \rightarrow 14^{c} \mathrm{j}$ & 7 & 59 & $\begin{array}{l}33 \\
34 \\
35 \\
36 \\
37\end{array}$ & $\begin{array}{l}\text { Filipino } 2 \\
\text { Hilda } 153 \\
\text { Hilds matina } \\
\text { Polar circle } \\
\text { Tuckcross } 756 \mathrm{P}\end{array}$ \\
\hline
\end{tabular}

$\left(^{*}\right)$ Les mesures n'ont pas été effectuées le $17^{\mathrm{e}}$ jour.

Nous classons ensuite 4 variétés d'Europe du Nord ou du Canada : "Freiland früheste », «Avon Early ", "Outdoor Girl » et «Polar Circle », (lots $n^{0} 4,8,11$ et 36) qui se situent entre la moyenne des 2 témoins et «Précoce ». Elles ne semblent cependant pas d'un niveau de résistance suffisant pour être utilisées comme géniteurs de bonne fertilité au froid.

Le comportement comparable à «Précoce» de «Hilds matina " et d'une origine de $L$. pimpinellifolium « 108059 $2(5) »\left(\right.$ lots $n^{\circ} 35$ et 27 ) rend ces 2 variétés aussi intéressan- tes que "Précoce » comme géniteurs de bonne fertilité au froid. "Hilds matina " est une variété précoce à feuilles de pomme de terre, à petits fruits ronds lisses à 2-3 loges, ce qui peut laisser supposer une origine commune avec « Précoce ».

Quatre variétés seulement, sur les 37 testées, sont supérieures à «Précoce » d'après le test utilisé. Il s'agit de "Prachtlow» qui est de type cerasiforme (lot $\mathrm{n}^{\circ} 7$ ), des 2 origines de $L$. hirsutum d'altitude, "LA 1393" et «LA 1777 » (lots n ${ }^{\circ} 14$ et 15 ) et d'une sélection I.N.R.A. de 

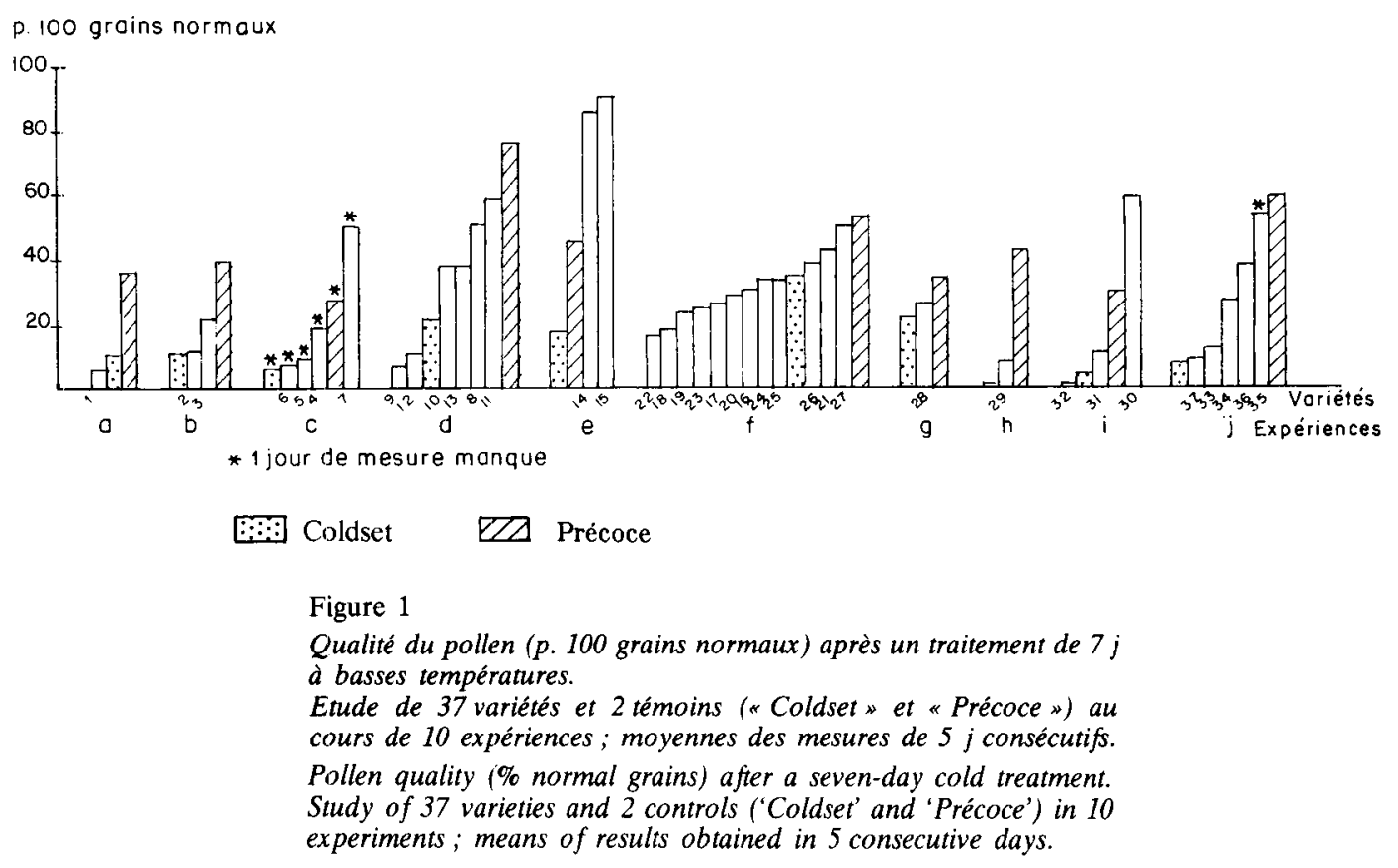

L. peruvianum (lot $\mathrm{n}^{\circ} 30$ ). Parmi ces 4 variétés intéressantes, le comportement de «LA 1393 » et de «LA 1777 » est tout à fait rernarquable; en effet lors de mesures non présentées ici, effectuées du $5^{\mathrm{e}}$ au $17^{\mathrm{e}}$ jour après le traitement à $7{ }^{\circ} \mathrm{C}$, nous n'avons observé aucun effet du froid sur le pollen : toutes les mesures étaient supérieures à 79 p. 100 chez "LA 1393 " et supérieures à 82 p. 100 chez «LA 1777 ». La bonne adaptation au froid de « LA 1777 » avait déjà été signalée pour la germination du pollen (ZAMIR et al., 1981) et l'origine géographique de ces lots de L. hirsutum (haute altitude) peut expliquer cette résistance au froid.

\section{CONCLUSIONS}

Sur 37 variétés testées pour l'aptitude à former du pollen au froid, 6 seulement sont de niveau voisin ou supérieur au témoin de résistance au froid «Précoce » : « Hilds matina », la lignée «108059-2 (5)" de L. pimpinellifolium, "Prachtlow », une sélection I.N.R.A. de L. peruvianum et 2 origines de L. hirsutum (« LA 1393 » et « LA $1777 »)$ ).
A ces 6 variétés intéressantes pour leur bonne microsporogenèse à $7{ }^{\circ} \mathrm{C}$, nous pouvons ajouter «Immuna Prior Beta » signalée précédemment (MAISONNEUVE, 1983) ainsi que les 7 variétés repérées en serre froide pour leur bonne production de pollen qui ont pu être rattachées à 2 grands groupes, les variétés du type «Précoce " et les variétés du type "Marmande» (Maisonneuve \& Philouze, 1982).

Nous ne disposons donc pas d'une grande variabilité, dans l'espèce $L$. esculentum, parmi les géniteurs de bonne fertilité pollinique au froid. Parmi ces variétés, “LA 1393 » et « LA 1777 » sont les meilleurs géniteurs de résistance au froid, puisqu'aucun effet du traitement n'a été décelé. Ainsi, malgré les problèmes liés à l'utilisation des espèces sauvages (relative difficulté d'obtention des $F_{2}$, nombre de générations à faire avant d'introduire les caractères intéressants dans une plante de type $L$. esculentum), ces 2 lots semblent les plus prometteurs pour améliorer la nouaison de la tomate à basses températures.

Reçu le 30 novembre 1982. Accepté le 9 mai 1983.

\section{RÉFÉRENCES BIBLIOGRAPHIQUES}

El Ahmadi A. B., Stevens M. A., 1979. Reproductive responses of heat-tolerant tomatoes to high temperatures. J. am. Soc. hortic. Sci., 104 (5), 686-691.

Graham T. O., 1961. Nagcarlang tomato type from the Philippines. Rep. Tomato Genet. Coop., 11, 40-41.

Harris R. E., 1972. Three new Sub-Arctic type tomatoes : « Early Sub Arctic », "Sub Arctic Midi » and «Sub Arctic Plenty ». Can. J. Plant Sci., 52, 119-120.

Harris R. E., 1976. Breeding tomatoes for the sub-arctic regions. Can. Agric., 21 (1), 27-29.

Maisonneuve B., 1983. Qualité du pollen formé au froid chez la tomate (Lycopersicon esculentum Mill.). I. Amélioration d'un test en conditions contrôlées. Agronomie, 3 (9), 873-878.

Maisonneuve B., Philouze J., 1982. Action des basses températures nocturnes sur une collection variétale de tomate (Lycopersicon esculentum Mill.). II. Etude de la quantité et de la qualité du pollen. Agronomie, 2 (5), 453-458.
Robinson R. W., Mishanec W., Shannon S., 1966. Fruit setting ability in relation to extreme temperatures. Rep. Tomato Genet. Coop., 16, 33.

Rudich J., Zamski E., Regev Y., 1977. Genotypic variation for sensitivity to high temperatures in the tomato: pollination and fruit set. Bot. Gaz., $138(4), 448-452$.

Trinklein D. H., Lambeth V. N., 1981. « Tuckcross 756 P 》 tomato. Hortscience, 16 (2), 230-231.

Zamir D., Tanksley S. D., Jones R. A., 1981. Low temperature effect on selective fertilization by pollen mixtures of wild and cultivated tomato species. Theor. appl. Genet., 59, 235-238. 